\title{
A Prototype of a High-Resolution Computerized Radiology Teaching File
}

\author{
Peter L. Apicella, G. James Blaine, and R. Gilbert Jost
}

\begin{abstract}
Computers provide an excellent tool for handling the task of organizing a radiology teaching file. Currently available computerized teaching files are either filmbased, slide-based, or use laser-disc video technology for image display. There are obvious advantages to having the management of radiologic images under the control of a computer, and the need for a higher resolution alternative to video laser-disc technology becomes apparent when one tries to computerize a chest radiology teaching file. We describe the prototype of such a system, named MIRTLE, (the Mallinckrodt Institute of Radiology Teaching and Learning Environment) which was designed to integrate text under the control of a custom data base with highresolution digital images from a Picture Archiving and Communications System. This system with its easy-touse windows environment should allow a significant increase in the use of the radiology teaching file.

Copyright 11991 by W.B. Saunders Company
\end{abstract}

KEY WORDS: education, radiology, computer, teaching file, PACS, digital images, data base.

F ELSON HAS noted, "Information is only as valuable as it is accessible, and the more information you have, the less accessible it is." In 1972, he described an early electromechanical radiology teaching file that used punched cards and a mechanical sorter. In the late $1970 \mathrm{~s}$, Levy used a computed tomography (CT) scanner to maintain an active teaching file that could display CT images. ${ }^{2}$ Smith and Rowberg later expanded that system to include a database which could manage information and allow searching for cases of interest. ${ }^{3}$ More recently, as the personal computer became commonplace, additional computerized teaching files have been reported, ${ }^{46}$ all of which use a commercial data base for information storage and retrieval. Arenson, in 1986, discussed the future of such systems and elaborated on the potential use of laser-disc video technology for image storage and display. Although the image quality was not adequate for interpretation of many forms of radiology, the interactive video provided a unique and beneficial learning environment. He alluded to the day when highresolution digital images might be integrated into a fully computerized radiology teaching file. $^{7}$

The goal of MIRTLE, the Mallinckrodt Insti- tute of Radiology Teaching and Learning Environment, is to be a general purpose educational package, a teaching engine, which integrates text and high-resolution digital images in a windowed environment. In its conceptual design, the teaching engine is an integrated data base of text and images that can be instructed without requiring knowledge of computer programming to teach any subject that requires reading text and viewing images as part of the educational process. Fields in which such a tool would be of particular use include radiology, pathology, and anatomy. The MIRTLE prototype has centered around the design of a custom chest radiology teaching file.

Presently, several computerized teaching file systems are available for radiologists. Systems such as Sommer's ${ }^{5}$ and Sistrom's ${ }^{6}$ use a commercial data base program to maintain information on an existing film-based library. Others make use of a computer monitor for text display and laser-disc video technology for television-quality image display. This includes the commercial computerized American College of Radiology (ACR) teaching file (Reston, VI). For portions of radiology this is an acceptable learning environment, for example CT and magnetic resonance imaging (MRI), but higher resolution than this system can provide is required to ideally visualize radiologic images such as chest and bone films.

The text storage software used by available computerized radiology teaching files is typically a general purpose database program such as dBase IV (Ashton Tate, Inc, Culver City, CA). This has the advantage of being easy to set up and design but has limits both in searching flexibility and speed. A teaching engine such as MIRTLE requires a database that is faster than most that are commercially available. Incorpo-

From the Mallinckrodt Institute of Radiology and the Washington University School of Medicine, St Louis, MO.

Address reprint requests to $R$. Gilbert Jost, MD, Mallinckrodt Institute of Radiology, St Louis, MO 63110.

Copyright $\odot 1991$ by W.B. Saunders Company

0897-1889/91/0401-0004\$03.00/0 
rated into its design, therefore, is a customized database that facilitates the rapid searching of key word phrases across multiple subjects.

\section{MATERIALS AND METHODS}

The computer used for this prototype is a Digital Equipment Corporation (DEC, Maynard, MA) VAXstation 3100 with 28 megabytes internal memory and 300 megabytes winchester disk storage. The system can be operated in a stand-alone fashion; however, in this implementation it was linked to the Mallinckrodt Institute of Radiology Information and Image Management System. The VAXstation supports a graphics monitor that allows text to be displayed in a windows-based environment. It also has the ability to display small, good quality versions of true radiographic images (image icons), which can be used for rapid case identification and image selection (Fig 1).

A custom Picture Archiving and Communications System library (PACSlib) written and maintained by the Mallinckrodt Institute of Radiology, Electronic Radiology Laboratory, was used for image storage and retrieval. ${ }^{8}$ A small prototype teaching image data base was created as separate entity from the Institute's clinical image data base of active cases. These data bases are connected so that images can be imported directly from the clinical data base into the teaching data base, thus allowing interesting cases to be quickly and easily added to the teaching file.

The PACS design enables images to Jeside both locally and across a netwotk on any storage media (magnetic disk, tape, or optical disk) in any resolution. Available digital images include CT $(512 \times 512 \times 12$ bits, 4,096 shades of grey), MRI ( $256 \times 256 \times 12$ bits), and portable chest films $(1,024 \times 1,024 \times 10$ bits, 1,024 shades of grey, scaled from $2,140 \times 1,740 \times 10$ bits acquired from a Philips Computed Radiography [PCR] 901 Storage Phosphor System). Future integration of film scanning equipment will allow images to be acquired from an existing teaching file as well.
Digital images were displayed on an ImLogix 1,000 monitor $(1,024 \times 1,024 \times 12$ bits, with 256 shades of grey $)$ with dynamic window, centering, and zoom capabilities. Future versions of MIRTLF, will be able to use higher resolution display devices including the Megascan display $(2,500 \times 2,048 \times 12$ bits $)$.

The software component of MIRTLE was written in C and relies on DECwindows (Digital Equipment Corporation, Maynard, MA), an implementation of $\mathrm{X}$ Windows (Massachusetts Institute of Technology), a networkoriented multitasking window environment. The data base was built using the VAX Record Management Service (RMS) file system and consists of a custom indexing scheme, which allows individual words or phrases to be grouped by subject and searched rapidly in a B-tree style." Both free-format text and key words are stored with each case as well as maintained in indexes.

\section{RESULTS AND DISCUSSION}

The prototype of MIRTLE was designed to show the advantages of combining digital images with resolutions higher than video-quality and a rapid-access database to locate cases in a teaching file. In order to show the use of the teaching engine concept, a multiple-choice quiz module was also integrated into the system.

\section{The Data Base}

The radiology teaching file implementation requires some definitions: a "case" is a collection of studies which describe a particular teaching point (eg, pneumonia or John Smith's films); a "study" is a collection of images that make up a particular radiologic examination (eg, poste-

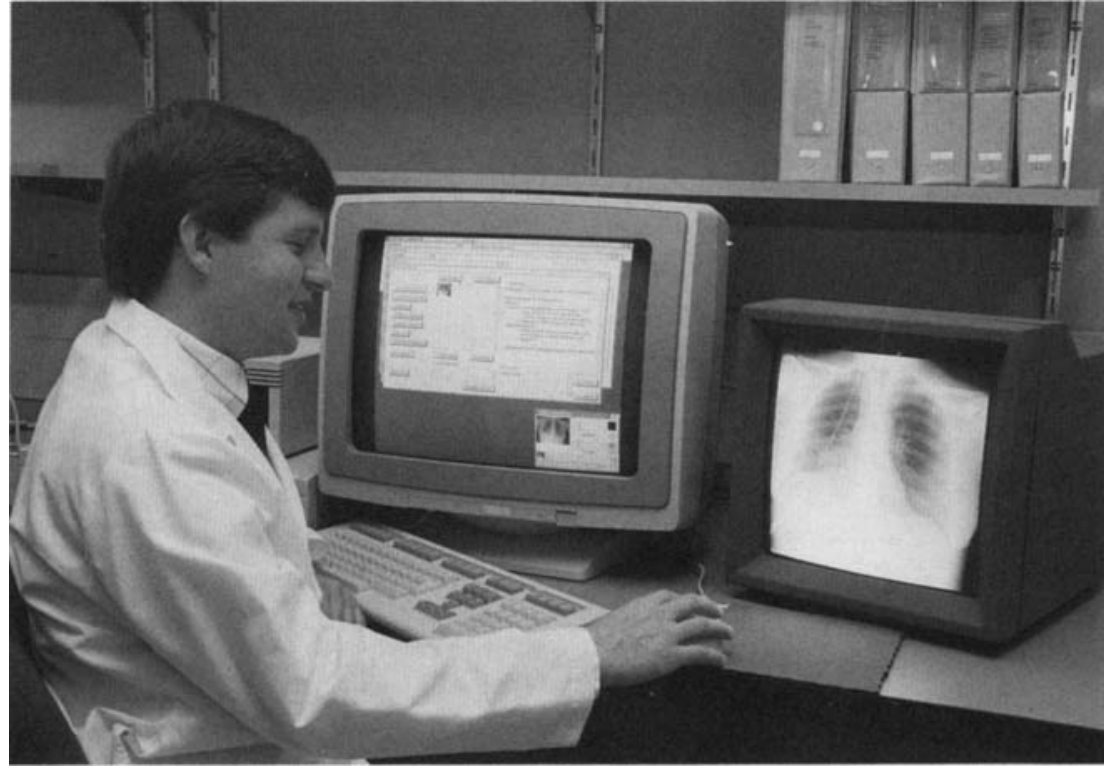

Fig 1. The computerized radiology teaching file, MIRTLE. has a display that shows text and small image icons. The adjacent high-resolution monitor shows quality radiologic images for interpretation. 
rior-anterior [PA] and lateral [LAT] chest films make up a set of chest films); and an "image" is what is actually displayed on the monitor for interpretation (Fig 2). Each case may have multiple studies, each of which may have multiple images.

In concept, each case will have personal information (patient name, record number, age, and sex) and may have multiple diagnoses, multiple radiologic findings from different anatomic locations, and multiple film types. For this reason, a flexible database was designed that allows both free-format text and multiple key word phrases to be associated with each case.

As an example, a case of mesothelioma from patient John Smith, age 60, record number 12345 , would be classified by his diagnosis of mesothelioma and asbestosis as well as the findings on chest film of pleural plaques, an effusion, interstitial fibrosis, and a pleural mass. An additional CT finding of rib destruction would be specified. A case discussion could include the relative frequency of the radiologic findings of mesothelioma and asbestosis and the association between asbestos exposure and mesothelioma. Because this case would need to be classified under multiple diagnoses, multiple findings, and multiple film types, one can see that in a large teaching file, searching for information could be very time-consuming. MIRTLE contains a custom data base that allows the case to be identified by searching for any one or combination of the following key word examples: mesothelioma; mesothelioma and asbestosis; CT films, rib destruction, and mesothelioma. However, more important than flexibility in this or any system is the rapid speed at which a case can be identified. MIRTLE'S custom database allows case identification to be completed within a few seconds, even for very large data bases.

\section{The User Environment}

Another desirable feature of any teaching file is its ease of use by novices. A windowing environment, similar to the Macintosh Computer (Apple Computer, Inc., Cupertino, CA), was chosen as an effective user interface for MIRTLE. A hierarchy of windows allows the user to rapidly perform the desired tasks without requiring memorization of complicated commands.

To demonstrate how a case is located, a typical MIRTLE session will be discussed. An initial window presents the user with a choice of searching subjects (eg, by diagnosis, by radiologic finding, or by ACR code number). When a subject is selected, a list of available key words is

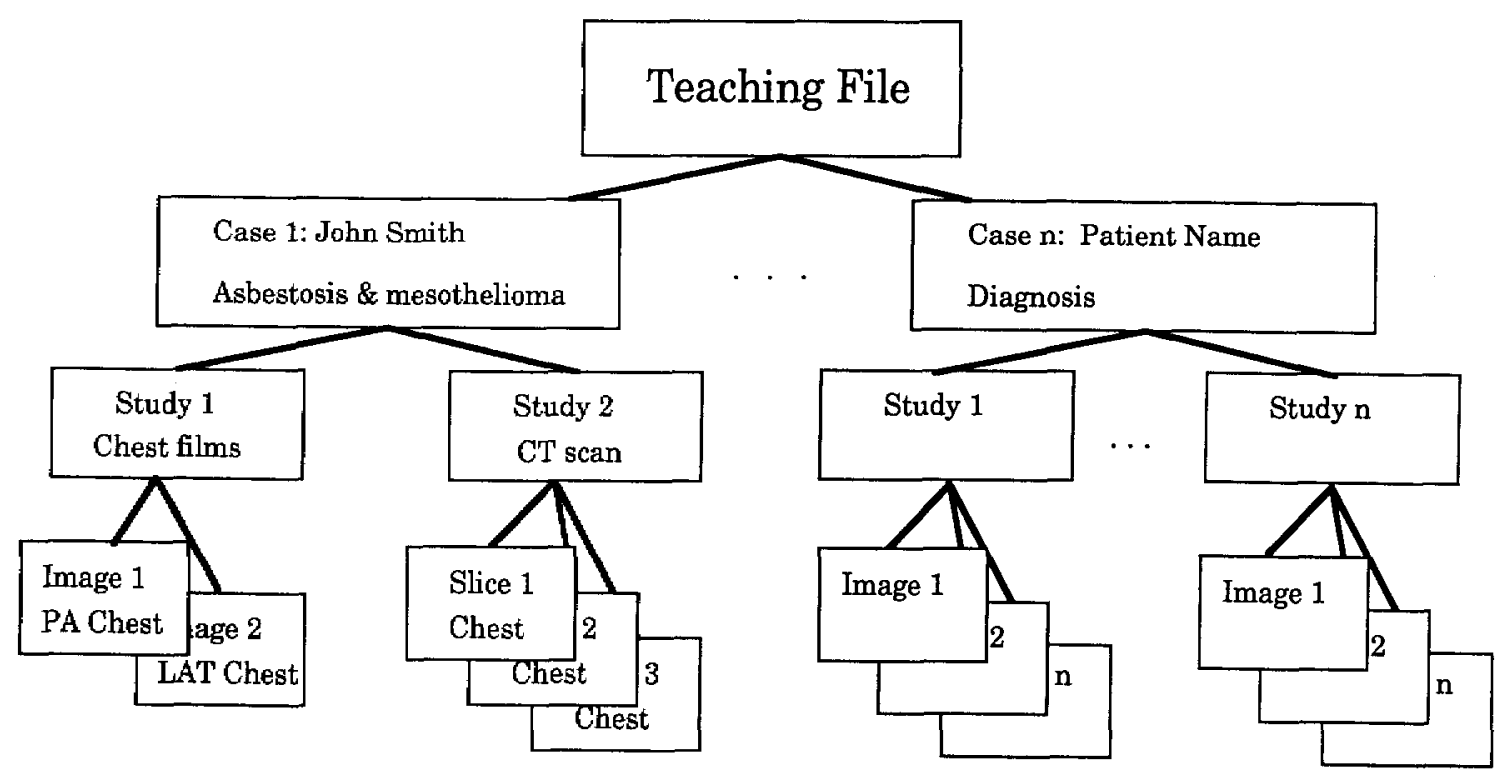

Fig 2. The data base hierarchy for the teaching file allows grouping of information by cases. Each case may be composed of a number of studies, each of which may have several images. These images can be displayed on a high-resolution monitor. Each case is referenced using a B-tree style index. 
shown. An item can be selected directly from this list, or it can be entered from the keyboard. Once selected, the number of cases in the teaching file associated with that key word is displayed (Fig 3). Multiple subjects and key words can be chosen to limit the search. As each key word is added to the search list, the actual number of cases matching all criteria is displayed. Items can be added or deleted from the search list to reduce the time required to identify a specific case. Rapid user feedback is especially important in this process. MIRTLE's custom database provides immediate (less than 1 second) responses to multiple searches (similar searching using $\mathrm{dBase}$ III on an IBM PC compatible computer with a database of 400 cases took about 10 seconds for one key word and would be significantly longer for more than one key word). Custom databases such as those used in this prototype offer the advantage of rapid access, but we recognize that commercial database packages offer important advantages in terms of supportability and error recovery. Once a list of search criteria has been specified, the matching list of cases is presented, each with a short description, film type, and a small image icon that aids in identification (Fig 4).

When a specific case is selected, a window containing text and the key words of the case is presented (Fig 5). The information is grouped by subject and can be unlimited in length. Numerous key words can be specified for each case, and information can be updated at any time. In addition to text information, a list of film studies and small image icons that are reduced versions of actual digital images is displayed.

\section{The Digital Images}

To view an image in full size on the separate monitor, the image icon is selected. Currently, approximately 10 seconds is required to display a $256 \times 256 \times 12$ bit image (MRI films) on the ImLogix 1000 monitor $(1,024 \times 1,024 \times 12$ bits $)$. These images can be paneled $4 \times 4$ and zoomed dynamically. An image $1,024 \times 1,024 \times 10$ bits (chest film) requires approximately 1 minute to display. These retrieval times, while acceptable for a prototype demonstration, do not match the performance of video-disc based systems. The time constraints for our digital system must be reduced for the system to be a truly useful teaching tool, and work is in progress to accomplish this goal.

Image manipulation capabilities include dynamic window and centering (grey scale adjust-

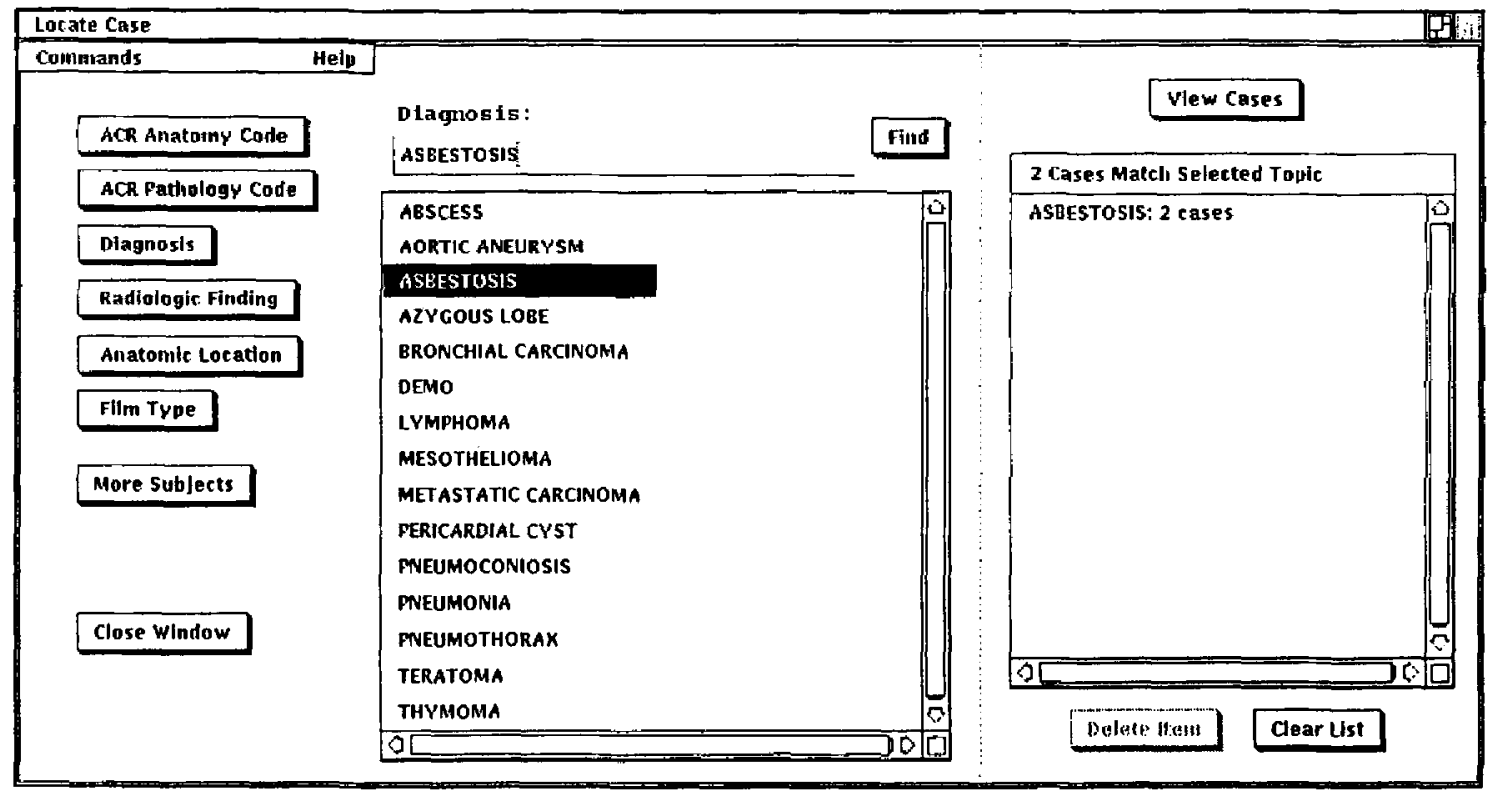

Fig 3. To locate a case of interest, a topic (eg, diagnosis) is selected. An alphabetical key word list is then displayed. The item asbestosis has been selected in this example, and two matching cases are quickly located. 
Fig 4. Each matching case is identifled by a small image icon, a description, and an identification number. This allows rapid identification of desired cases when a large number of matehes exist for a key word.

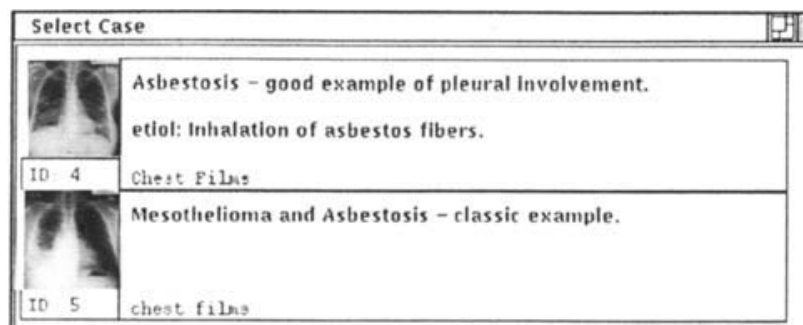

ment), bilinear interpolated zooming (entarging the image) from any center, and cine loop (movie-style image display). CT and MR images can be placed in any location on the monitor with any ordering.

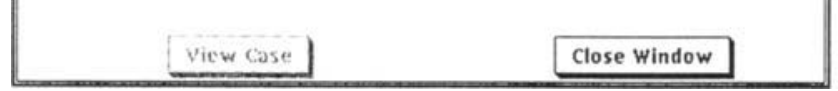

The preference for image resolution beyond that of video-disc quality for a chest teaching file is apparent when one compares a video-disc image and MIRTLE's high-resolution monitor to identify a case study such as a pneumothorax.

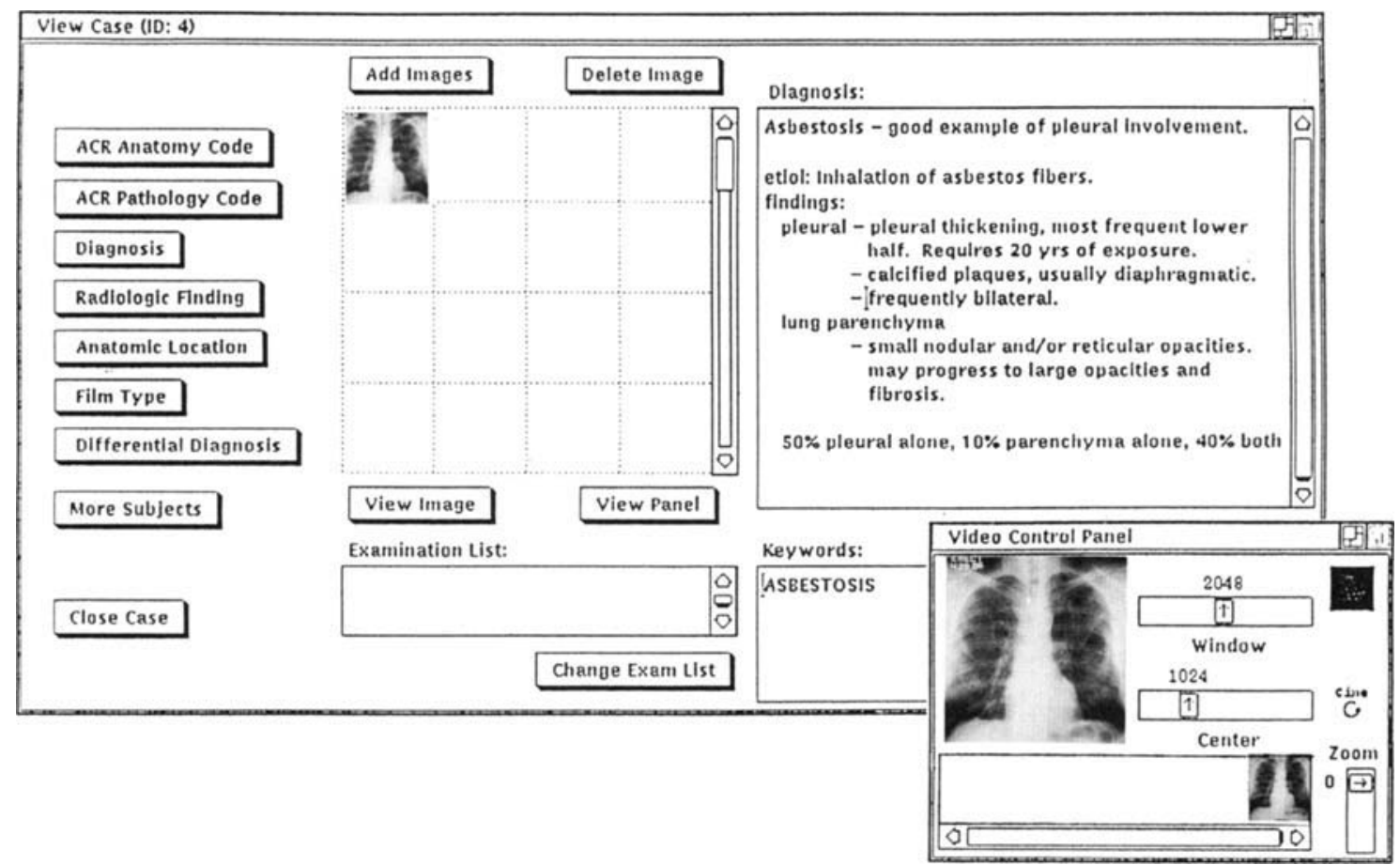

Fig 5. The desired case has been selected and information on the case is displayed. The chest film can be selected for display on a high-resolution monitor, and the video control panel allows image manipulation including grev-scale adjustments. 
MIRTLE allows images to be obtained at varying resolutions so that pertinent details of a the teaching file image can be readily discerned.

\section{The American College of Radiology Codes}

Because the American College of Radiology (ACR) Index for Radiologic Diagnosis codes are a useful way of organizing a teaching file, an electronic version of the revised third edition was included in MIRTLE. ${ }^{10} \mathrm{~A}$ utility was written to format the ACR codes into an expandable outline format. If the ACR Pathology or ACR Anatomy subject is selected as a search topic, the user is presented with the 10 body sections of the ACR code. When a body section is selected, it will expand so that all items with one additional digit are shown in a list (Fig 6). If additional subclassification is necessary, a subitem can be selected, and it will expand. This process can be repeated until the desired ACR code number is located. An item can be both expanded and closed to aid in rapidly identifying the desired code. This format works very well in the windowed environment of MIRTLE, and may prove an attractive method in other applications that use an electronic version of the ACR codes.

Once a code number is selected, it can be used as a key word to search the database. In addition, wildcard searches can be performed using an asterisk $\left({ }^{*}\right)$. For example, in the body section lung, mediastinum and pleura, .214 is an aspiration pneumonia, .21* can be used for any pneumonia or abscess and would include 210 to .219 .

\section{The Teaching Engine Concept}

In order to demonstrate the flexibility of the teaching engine design of MIRTLE, a multiple choice quiz module was added to the system. It functions simply to show text and images (which have been selected from an existing case using the previously mentioned procedures) along with response choices $A$ to $D$. Selecting an individual response will display a window that can contain text or additional images and will control flow to additional windows ( $\mathrm{Fig} 7$ ). The images can be viewed in high-resolution with grey-scale adjustment and zooming. From this simple design, a complicated teaching lesson can be created by someone who has no computer programming knowledge. It can be maintained and modified as necessary.

Additional modules to be added to MIRTLE might include a slide-show style presentation of cases for lectures. This could include handdrawn pictures and charts that would be scanned into the computer. Another module might include a general purpose quiz for medical stu-

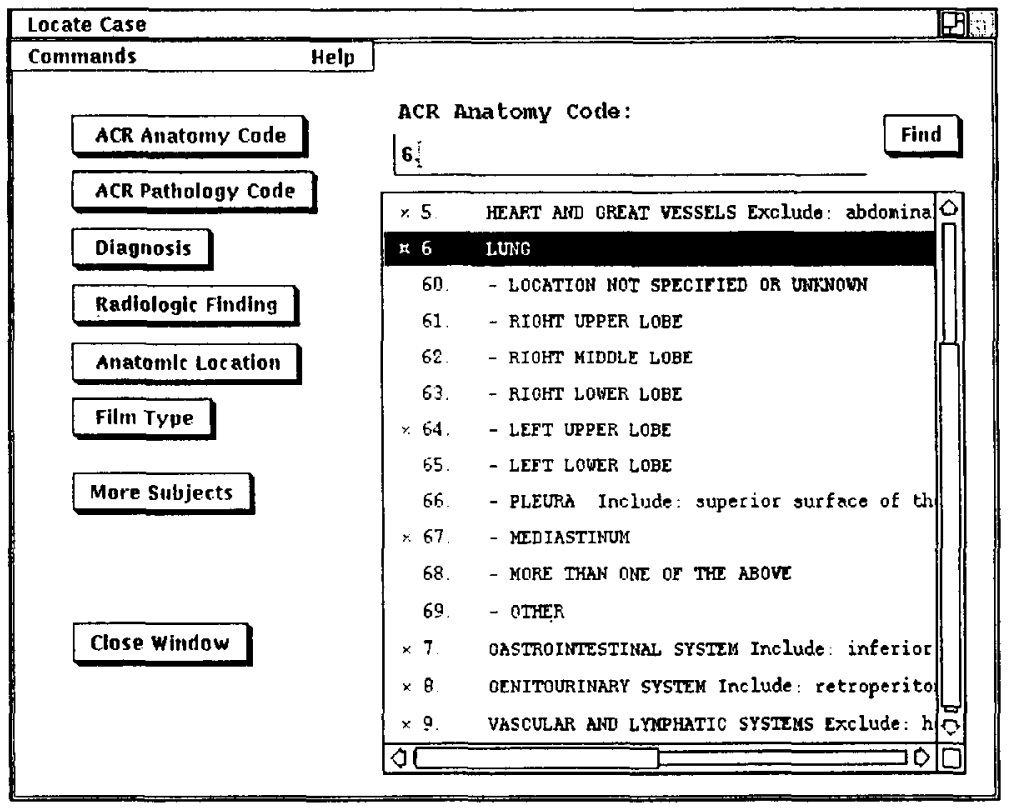

Fig 6. To locate a case by the ACR anatomic code number, the desired body section is selected. That section expands like an outline to show more specific choices. The actual numeric coding is done automatically. 


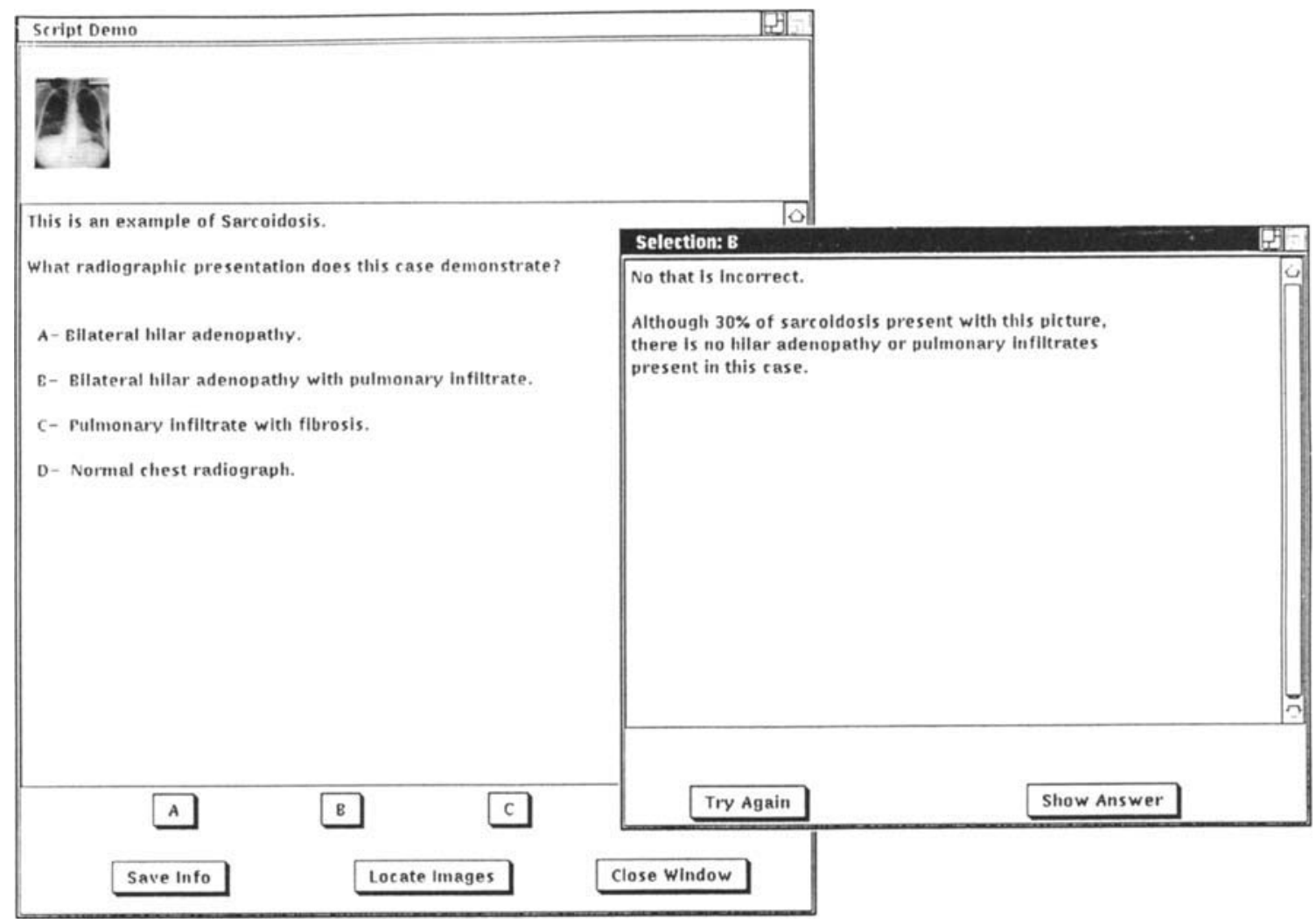

Fig 7. The quiz module provides both text and image icons. Full images can be displayed on the high-resolution monitor by selecting the icons. Responses to the question are multiple choice: A to D. Here, an incorrect answer has been selected, and the user can try again or request the correct answer.

dents and residents, which could be automatically tailored to the abilities of its user and test areas more thoroughly that are determined to be deficient.

\section{The Future}

Certainly, computers have come a long way over the past 2 decades since Felson's electromechanical teaching file. In the future as highresolution displays are integrated into radiology departments for routine image interpretation, the computerized teaching file is sure to follow. MIRTLE provides an early look into this future and, although much work remains to be done, it shows the advantages of both a high-resolution display and a fast, custom-designed database for a radiology teaching file.

\section{ACKNOWLEDGMENT}

Our thanks to Stephen Moore for his guidance and assistance with DECwindows, Robert Whitman for image acquisition and display, and James Studt as designer of PACSlib. Their efforts in creating the PACS testbed within the Electronic Radiology Laboratory facilitated development of the project. Special thanks to Dr Dixie Anderson for her inspiration and advice and to Dr Ronald Evens, Director of the Mallinckrodt Institute of Radiology, for his support of the project.

\section{REFERENCES}

1. Felson B, Feltham C: A simple method of information retrieval. Radiology 105:315-318, 1972

2. Levy JM: The use of a CT scan computer for non-CT applications: Teaching file program. Radiology 131:530-531, 1979

3. Smith DF, Rowberg AA: Data-base management system on a CT scanner computer: Application to teaching fite and procedure records. AJR 142:1219-1223, 1984

4. Garra BS, Kurtz D, Shawker TH, et al: A teaching file program for use in an ultrasound department. J Ultrasound Med 5:313-317, 1986

5. Sommer FG: Some applications for a personal com- 
puter data-base system in radiology. AJR 147:1075-1077, 1986

6. Sistrom CL, Black WC: A computerized filing system for radiology teaching cases. AJR 149:191-193, 1987

7. Arenson RL: Teaching with computers. Radiol Clin North Am 24:97-103, 1986

8. Jost RG, Wessel W, Blaine GJ, et al: PACS-Is there light at the end of the tunnel? Proceedings of Society of Photo-optical Instrumentation Engineers-Medical Imaging III 1093:74-84, 1989

9. Knuth DE: The Art of Computer Programming: Sorting and Searching, vol 3. Reading, MA, Addison-Wesley, 1973

10. Index for Radiologic Diagnosis, Revised 3rd Edition. Reston, VI, American College of Radiology, 1986 